Research Paper

\title{
Impact of the cultivation strategy on resveratrol production from glucose in engineered Corynebacterium glutamicum
}

\author{
Adelaide Braga $^{\mathrm{a}, \mathrm{b}, *, 1}$, Joana Oliveira ${ }^{\mathrm{a}, \mathrm{b}, 1}$, Rita Silva ${ }^{\mathrm{a}, \mathrm{b}}$, Patricia Ferreira ${ }^{\mathrm{a}, \mathrm{b}}$, Isabel Rocha ${ }^{\mathrm{a}, \mathrm{b}}$, \\ Nicolai Kallscheuer ${ }^{c}$, Jan Marienhagen ${ }^{c}$, Nuno Faria ${ }^{a, b}$ \\ ${ }^{a}$ Biotempo, Avepark - Edif. Spinpark, Zona Industrial da Gandra, Barco, 4805-017, Guimarães, Portugal \\ ${ }^{\mathrm{b}}$ Centre of Biological Engineering, University of Minho, Campus de Gualtar, 4710-057, Braga, Portugal \\ ${ }^{\mathrm{c}}$ Institute of Bio- and Geosciences, IBG-1: Biotechnology, Forschungszentrum Jülich, 52425, Jülich, Germany
}

\section{A R T I C L E I N F O}

\section{Keywords:}

Batch

Corynebacterium glutamicum

Fed-batch

Oxidation

Resveratrol

\begin{abstract}
A B S T R A C T
The health benefits of polyphenols such as stilbenes and flavonoids for humans are increasingly attracting attention. Resveratrol is a well-characterized naturally-occurring stilbene and potent anti-oxidant, which is used as food supplement and cosmetic ingredient. Several microorganisms including Corynebacterium glutamicum were engineered for resveratrol production from glucose. Based on the cultivation of a resveratrol-producing $C$. glutamicum strain in shake flasks, different strategies for improving production under controlled conditions at bioreactor scale were tested. To this end, different cultivation parameters including substrate concentration and operation modes (batch and fed-batch) were evaluated. Whereas the highest biomass concentration was observed during fed-batch fermentation, the maximum resveratrol production was achieved in batch mode. The maximal titer obtained was $12 \mathrm{mg} \mathrm{L}^{-1}$ of resveratrol without the addition of the fatty acid synthase inhibitor cerulenin, which was previously shown to be crucial for production with $C$. glutamicum. The specific growth rate during production seems to have a significant effect in resveratrol production and apparently low specific growth rates may redirect the metabolic bottleneck from $p$-coumaric acid formation to malonyl-CoA or ATP availability. We also show that high oxygen concentrations in the bioreactor negatively affected the obtained resveratrol titers with $C$. glutamicum, which is most likely due to the strong tendency of resveratrol to oxidize or oligomerize. Thus, up-scaling of the resveratrol production process is technically challenging and individual process parameters have to be optimized cautiously.
\end{abstract}

\section{Introduction}

Resveratrol (3,5,4'-trihydroxystilbene) is a well-characterized plantderived polyphenol, which is commonly used as a nutritional supplement and medicinal ingredient (Baur and Sinclair, 2006). This compound from the stilbene family is present in berry fruits, such as grapes, blackberries, blueberries and raspberries (Kiselev, 2011). The consumption of food rich in these compounds improves the anti-inflammatory and anti-oxidative stress response and can help to prevent cancer, obesity and chronic diseases, such as diabetes, neurodegenerative and cardiovascular diseases (Manach et al., 2004). Due to the health-promoting properties, the demand for resveratrol is expected to further increase in the future. Nowadays, commercially available resveratrol is mostly obtained by extraction from plants, such as Polygonum cuspidatum (Lin et al., 2016). However, this procedure has several drawbacks since the natural amount of resveratrol in plants varies depending on environmental and geographic conditions. In particular, stilbenes such as resveratrol are not produced constitutively, but their biosynthesis is often induced as metabolic response to infections (Bavaresco et al., 1997). Also, the purification of individual polyphenols from plant material is technically challenging, as the natural concentrations are low and extracts typically contain a variety of chemically very similar polyphenolic compounds (Marienhagen and Bott, 2013).

In contrast, biotechnological production of resveratrol by genetically engineered microorganisms represents a promising alternative to extraction from plants. In recent years, microbial resveratrol production was achieved in yeast and bacteria by the introduction of the heterologous pathway from plants and engineering of the microbial host metabolism towards increased production (Beekwilder et al., 2006; Li et al., 2015; Vos et al., 2015). In the constructed strains, resveratrol is typically produced via three enzymatic steps starting from the aromatic

\footnotetext{
* Corresponding author.

E-mail address: abraga@deb.uminho.pt (A. Braga).

${ }^{1}$ These authors contributed equally to this work.
} 


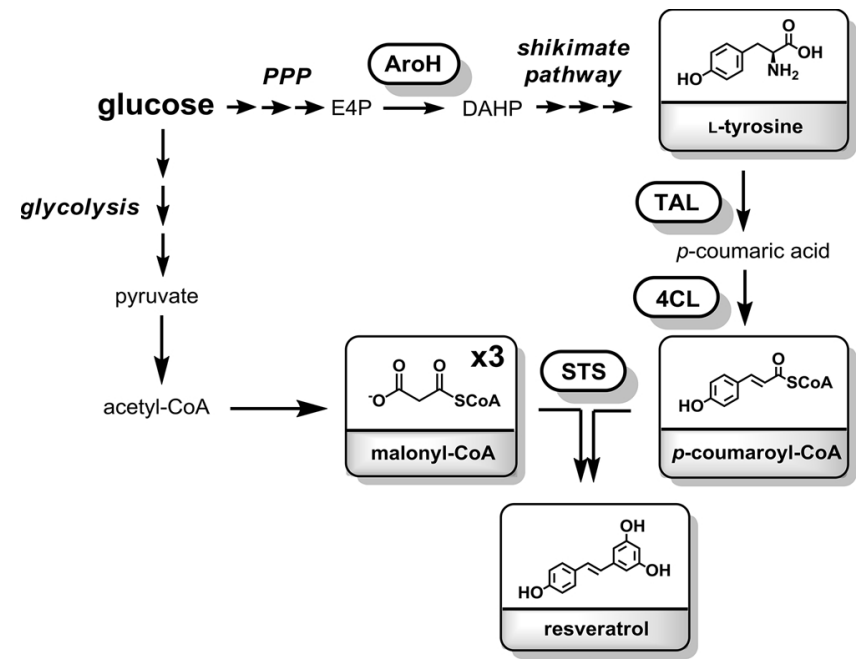

Fig. 1. Schematic representation of the overall pathway for resveratrol production. Abbreviations: E4P: erythrose 4-phosphate, DAHP: 3-deoxy-D-arabinoheptulosonate 7phosphate, AroH: DAHP synthase (encoded by the gene aroH), TAL: tyrosine ammonia lyase, 4CL: 4-coumarate: CoA ligase, STS: stilbene synthase, PPP: pentose phosphate pathway.

amino acid 1-tyrosine. The respective reactions are catalyzed by tyrosine ammonia lyase (TAL), 4-coumarate: CoA ligase (4CL) and stilbene synthase (STS) (Fig. 1). The TAL non-oxidatively deaminates l-tyrosine to $p$-coumaric acid, which is then converted to the respective CoA thioester $p$-coumaroyl-CoA by 4CL. In the third step, three molecules of malonyl-CoA are condensed with one molecule of 4-coumaroyl-CoA by STS yielding one molecule of resveratrol. For microbial resveratrol production from glucose, the pentose phosphate pathway and the shikimate pathway were additionally engineered to provide sufficient amounts of the resveratrol precursor, l-tyrosine (Fig. 1) (Vos et al., 2015).

The first studies reported the use of Saccharomyces cerevisiae and Escherichia coli for microbial production of resveratrol. Often, the production depends on the addition of precursors, such as $p$-coumaric acid, 1-phenylalanine or l-tyrosine, which renders the production processes expensive for industrial applications (Lim et al., 2011; Wu et al., 2013). In addition, the fatty acid synthase inhibitor cerulenin is often added to ensure sufficient malonyl-CoA availability in the host organism (Lim et al., 2011; van Summeren-Wesenhagen and Marienhagen, 2015). The very high price of cerulenin and the inhibition of growth resulting from the depletion of fatty acids are the main constraints for using cerulenin in large-scale fermentation of polyphenol-producing $E$. coli and $S$. cerevisiae strains.

Until today, also other organisms such as Corynebacterium glutamicum and Lactococcus lactis proved to be promising host organisms for resveratrol production (Donnez et al., 2009; Kallscheuer et al., 2016b). C. glutamicum is of particular interest for such applications as it shows a pronounced resistance to aromatic compounds (Shen et al., 2012). However, before $C$. glutamicum could be used for the production of resveratrol, a degradation pathway for the precursor $p$-coumaric acid in this organism had to be identified and abolished (Kallscheuer et al., 2016a,b). The highest resveratrol titer reported with C. glutamicum in shake flasks so far is $158 \mathrm{mg} \mathrm{L}^{-1}$, but the production still depended on the supplementation of $p$-coumaric acid and cerulenin (Kallscheuer et al., 2016b). Additional strain engineering towards deregulation of the aromatic amino acids metabolism and an optimal connection of the heterologous pathways to the host metabolism enabled resveratrol production starting from glucose without any need for supplementation of precursor metabolites (Kallscheuer et al., 2016b).

Based on the previous results obtained with $C$. glutamicum in shake flasks, we here aim to develop different fermentation strategies to improve the production of resveratrol from glucose with this organism. Since growth and resveratrol production in defined cultivation medium occur simultaneously, different experimental conditions (operation mode, oxygen supply and substrate concentration) were optimized in order to evaluate their impact on the resveratrol production capabilities of the engineered C. glutamicum strain.

\section{Experimental procedures}

\subsection{Bacterial strains, media and growth conditions}

The resveratrol-producing strain C. glutamicum DelAro ${ }^{4}$

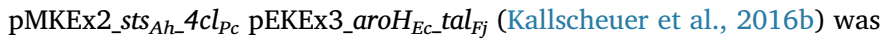
cultivated aerobically at $30{ }^{\circ} \mathrm{C}$ in Brain Heart Infusion (BHI) medium (only for precultures) or defined CGXII medium with glucose as sole carbon and energy source (Keilhauer et al., 1993). CGXII medium (pH

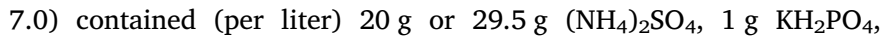
$1 \mathrm{~g} \mathrm{~K}_{2} \mathrm{HPO}_{4}, \quad 0.25 \mathrm{~g} \quad \mathrm{MgSO}_{4} \times 7 \mathrm{H}_{2} \mathrm{O}, \quad 10 \mathrm{mg} \quad \mathrm{CaCl}_{2}, \quad 10 \mathrm{mg}$ $\mathrm{FeSO}_{4} \times 7 \mathrm{H}_{2} \mathrm{O}, 10 \mathrm{mg} \mathrm{MnSO}_{4} \times \mathrm{xH}_{2} \mathrm{O}, 1 \mathrm{mg}$ of $\mathrm{ZnSO}_{4} \times 7 \mathrm{H}_{2} \mathrm{O}, 0.2 \mathrm{mg}$ $\mathrm{CuSO}_{4}, 0.02 \mathrm{mg} \mathrm{NiCl} 2 \times 6 \mathrm{H}_{2} \mathrm{O}, 0.2 \mathrm{mg}$ biotin, $5 \mathrm{~g}$ of urea, $42 \mathrm{~g}$ of 3morpholinopropanesulfonic acid (MOPS), $40 \mathrm{~g}$ or $80 \mathrm{~g}$ of glucose, and $30 \mathrm{mg}$ protocatechuic acid. For plasmid maintenance kanamycin ( $25 \mathrm{mg} \mathrm{L}^{-1}$ ) and spectinomycin $\left(100 \mathrm{mg} \mathrm{L}^{-1}\right.$ ) were added to the medium. In the fermenter experiments, urea and MOPS were omitted as the $\mathrm{pH}$ was automatically controlled.

Corynebacterium glutamicum was cultivated for $8-10 \mathrm{~h}$ in a $500 \mathrm{~mL}$ baffled Erlenmeyer flask containing $50 \mathrm{~mL}$ of BHI medium on a rotary shaker at $200 \mathrm{rpm}$ (first preculture) and was subsequently inoculated into $100 \mathrm{~mL}$ CGXII medium in $1 \mathrm{~L}$ baffled Erlenmeyer flasks (second preculture). The starting $\mathrm{OD}_{600}$ for the second preculture was 1 . The second preculture was cultivated for $16-17 \mathrm{~h}$ on a rotary shaker at $200 \mathrm{rpm}$ and used to inoculate the fermenter to an initial $\mathrm{OD}_{600}$ of 1 . The heterologous gene expression was induced with $1 \mathrm{mM}$ IPTG at a biomass concentration of $2-3 \mathrm{~g} \mathrm{~L}^{-1}$. The fermentation experiments were performed in a 5-L stirred-tank bioreactor BIOSTAT ${ }^{\circ} \mathrm{B}-\mathrm{DCU}$ II (Sartorius Stedim, Germany). The operating volume for the fermentations was $2.5 \mathrm{~L}$ and the $\mathrm{pH}$ was automatically controlled at 7.0 by addition of $3 \mathrm{M} \mathrm{NaOH}$ and $3 \mathrm{M} \mathrm{HCl}$. The dissolved oxygen was kept above $30 \%$ of saturation by feedback control of the stirring speed from $650 \mathrm{rpm}$ until a maximum of $1500 \mathrm{rpm}$ and an air-flow rate from 1.8 vvm (volume air per volume medium and minute) to 4.8 vvm. Foam was disrupted by automatic addition of antifoaming agent Antifoam 204 (Sigma-Aldrich).

In fed-batch fermentations, the culture was fed with a concentrated solution containing $400 \mathrm{~g} \mathrm{~L}^{-1}$ glucose, $40 \mathrm{gL}^{-1}\left(\mathrm{NH}_{4}\right)_{2} \mathrm{SO}_{4}, 5 \mathrm{~g} \mathrm{~L}^{-1}$ $\mathrm{KH}_{2} \mathrm{PO}_{4}$ and $5 \mathrm{~g} \mathrm{~L}^{-1} \mathrm{~K}_{2} \mathrm{HPO}_{4}$ as soon as the glucose concentration in the batch medium fell below $15 \mathrm{~g} \mathrm{~L}^{-1}$. In the beginning of fed-batch and after $24 \mathrm{~h}$ of growth, concentrated solutions with $2.5 \mathrm{~g} \mathrm{~L}^{-1} \mathrm{MgSO}_{4}$ and $100 \mathrm{mg} \mathrm{L}^{-1} \mathrm{CaCl}_{2}$ were added to the fermenter in order to restore the concentration of both reagents in the fermentation medium. The feeding rate was increased stepwise based on the exponential feeding strategy defined by Eq. (1), where $F(t)$ is the flow rate of the feeding solution, $\mu$ the biomass specific growth rate $\left(\mathrm{h}^{-1}\right), Y_{X / S}$ the yield of biomass on substrate ( $g$ cell dry weight per g glucose), $X$ the biomass concentration $\left(\mathrm{g} \mathrm{L}^{-1}\right), V$ the working volume (L) and $S$ the substrate concentration in the feeding solution $\left(\mathrm{g} \mathrm{L}^{-1}\right)$.

$F(t)=\frac{\mu}{Y_{X / S}} \frac{X(t) V(t)}{S}$

A constant value of 0.4 was used for $Y_{X / S}$, which represents an approximation for the biomass yield, while the desired specific growth rates tested were $0.03 \mathrm{~h}^{-1}\left(F_{A}\right), 0.05 \mathrm{~h}^{-1}\left(F_{B}\right), 0.07 \mathrm{~h}^{-1}\left(F_{C}\right)$ and $0.1 \mathrm{~h}^{-1}\left(F_{D}\right)$. 


\subsection{Sampling and analytical methods}

Samples from the bioreactor were taken at regular intervals for the analysis of biomass concentration, glucose consumption and $p$-coumaric acid and resveratrol production. Biomass concentration was determined by measuring the optical densities of the cell cultures at a wavelength of $600 \mathrm{~nm}\left(\mathrm{OD}_{600}\right)$. The obtained values were converted to biomass dry weight using a calibration procedure. High-performance liquid chromatography for the quantification of glucose was performed in a JASCO system using a refractive index detector (RI-2031). The samples were analysed using an Aminex HPX-87H column from Bio-Rad, which was kept at $60{ }^{\circ} \mathrm{C} ; 10 \mathrm{mM} \mathrm{H}_{2} \mathrm{SO}_{4}$ was used as mobile phase with a flow rate of $0.5 \mathrm{~mL} \mathrm{~min}^{-1}$. Quantitative analysis of glucose was performed by injecting standards with known concentrations. The calibration curve was calculated using the peak areas of the RI detector for glucose.

For the quantification of p-coumaric acid and resveratrol, metabolite extracts from the cultivation broth were prepared according to Kallscheuer et al. (2016b) with some modifications. $1 \mathrm{~mL}$ of the culture broth, acidified at $\mathrm{pH} 2$ with $6 \mathrm{M} \mathrm{HCl}$, was mixed with $1 \mathrm{~mL}$ ethyl acetate and vigorously shaked for $2 \mathrm{~min}$ at room temperature. The suspension was centrifuged for $5 \mathrm{~min}$ at $8.000 \mathrm{~g}$ and the ethyl acetate layer $(600 \mu \mathrm{L})$ was transferred to a solvent-resistant tube. After evaporation of the ethyl acetate overnight, dried extracts were resuspended in $100 \mu \mathrm{L}$ of acetonitrile and directly used for ultra-high-performance liquid chromatography (UHPLC) analysis. Resveratrol and $p$-coumaric acid in the extracted samples were quantified using a SHIMADZU UHPLC system equipped with a diode array detector (SPD-M20A). LC separation was carried out with a Kinetex $1.7 \mathrm{u} \mathrm{C}_{18} 100 \AA$ pore size column ( $50 \mathrm{~mm}$ by $2.1 \mathrm{~mm}$ [internal diameter]; Phenomenex) at $50{ }^{\circ} \mathrm{C}$. For elution, $0.1 \%(\mathrm{v} / \mathrm{v})$ acetic acid (solvent $\mathrm{A})$ and acetonitrile supplemented with $0.1 \%(\mathrm{v} / \mathrm{v})$ acetic acid (solvent $\mathrm{B})$ were applied as the mobile phases at a flow rate of $0.5 \mathrm{~mL} \mathrm{~min}^{-1}$. A gradient was used, where the amount of solvent B was increased stepwise: minute 0-6: $10 \%$ to $30 \%$, minute $6-7: 30 \%$ to $50 \%$, minute $7-8: 50 \%$ to $100 \%$ and minute 8-8.5: $100 \%$ to $10 \%$. Obtained area values were linear up to metabolite concentrations of at least $250 \mathrm{mg} \mathrm{L}^{-1}$.

\section{Results and discussion}

\subsection{Batch fermentation with $40 \mathrm{gL}^{-1}$ glucose}

The strain C. glutamicum DelAro ${ }^{4} \quad$ pMKEx2__ts $_{A h \_} 4 c l_{P c}$ pEKEx3_aroH Ec $_{t} t a l_{F j}$ used here was shown to produce resveratrol from glucose in shaking flasks (Kallscheuer et al., 2016b), but the strain was never cultivated under controlled conditions in a bioreactor before. During the cultivation in batch mode with an initial glucose concentration of $40 \mathrm{~g} \mathrm{~L}^{-1}$, the strain reached a maximum biomass concentration of $18 \mathrm{~g} \mathrm{~L}^{-1}$ after $23 \mathrm{~h}$ and grew with a maximum specific growth rate of $0.23 \mathrm{~h}^{-1}$ (Fig. 2A). Under these conditions $Y_{X / S}$ was 0.45 ( $g$ cell dry weight per $\mathrm{g}$ glucose), which is in agreement with published data for this bacterium (Buchholz et al., 2013). IPTG was supplemented to the fermenter five hours after inoculation to induce heterologous gene expression required for resveratrol production. An increase in the resveratrol titer was observed during the exponential growth phase and no further increase was achieved during the stationary phase. The highest titer of $4 \mathrm{mg} \mathrm{L}^{-1}$ resveratrol was obtained $27 \mathrm{~h}$ after inoculation (Fig. 2B). Production of resveratrol requires three molecules of malonyl-CoA per molecule of $p$-coumaryl-CoA (Fig. 1). In C. glutamicum, malonyl-CoA is exclusively provided for the synthesis of fatty acids, which in turn are required for cell wall and cell membrane synthesis. Typically, malonyl-CoA synthesis decreases in the stationary phase (as fatty acids are predominately required during growth) (Larisch et al., 2007), which explains why the production of resveratrol did not continue. At this stage, $p$-coumaric acid is no longer consumed for resveratrol production, which results in the accumulation of $1 \mathrm{mg} \mathrm{L}^{-1} p$ coumaric acid in the last $15 \mathrm{~h}$ of cultivation (Fig. 2B). No $p$-coumaric acid accumulated in the exponential phase, which is a hint that this intermediate might represent the rate-limiting metabolite at this stage of production. This observation is in line with the previous finding that the activity of the tyrosine ammonia lyase represents the decisive bottleneck in the production pathway for resveratrol from glucose in $C$. glutamicum (Kallscheuer et al., 2016b).

Resveratrol production in C. glutamicum occurred simultaneously to biomass formation. In the late exponential phase, cell growth started to decelerate as a result of resource depletion in the medium. For an optimized resveratrol production two different strategies were tested in order to prolong the exponential growth phase. These include batch fermentation with a higher initial glucose concentration and fed-batch fermentation starting from $40 \mathrm{~g} \mathrm{~L}^{-1}$ glucose and continuous feeding of glucose and a nitrogen source.

\subsection{Batch fermentation with $80 \mathrm{gL}^{-1}$ glucose}

The effect of increasing the initial glucose concentration was studied by carrying out batch fermentations under the previously described conditions but with a higher initial glucose concentration of $80 \mathrm{~g} \mathrm{~L}^{-1}$. In order to maintain the carbon to nitrogen ratio $(\mathrm{C}: \mathrm{N})$ around 9 , the concentration of $\left(\mathrm{NH}_{4}\right)_{2} \mathrm{SO}_{4}$ was also proportionally increased in these experiments. A maximum biomass concentration of $37 \mathrm{~g} \mathrm{~L}^{-1}$ was obtained in CGXII medium with an initial concentration of $80 \mathrm{~g} \mathrm{~L}^{-1}$ glucose $37 \mathrm{~h}$ after inoculation (Fig. 2C), which is twice as high as in the batch fermentation with $40 \mathrm{~g} \mathrm{~L}^{-1}$ glucose. The cells grew with a maximum specific growth rate of $0.17 \mathrm{~h}^{-1}$, but an extended lag phase of around $8 \mathrm{~h}$ was observed when starting with $80 \mathrm{~g} \mathrm{~L}^{-1}$ glucose. Glucose was completely depleted after $32 \mathrm{~h}$. Under these conditions the biomass yield was 0.46 ( $g$ cell dry weight per $g$ glucose), which is comparable to the value obtained for the batch cultivation with $40 \mathrm{~g} \mathrm{~L}^{-1}$ glucose. After $37 \mathrm{~h}$ a maximal resveratrol concentration of $12 \mathrm{mg} \mathrm{L}^{-1}$ was observed, which is 3-fold higher compared to the batch cultivation with $40 \mathrm{~g} \mathrm{~L}^{-1}$ glucose (Fig. 2D). Obviously, the resveratrol titer is higher as more glucose was added to the medium. However, the yield (in mg resveratrol per $g$ glucose) of 0.15 (batch with $80 \mathrm{~g} \mathrm{~L}^{-1}$ glucose) was also slightly higher than the yield of 0.10 obtained for the batch fermentation with $40 \mathrm{~g} \mathrm{~L}^{-1}$ glucose. $p$-Coumaric acid did not significantly accumulate under these conditions $\left(0.48 \mathrm{mg} \mathrm{L}^{-1}\right)$.

\subsection{Fed-batch fermentation}

Fed-batch cultivation represents the most common technique for achieving a high specific productivity while avoiding oxygen limitation or substrate inhibition. Exponential feeding profiles are usually set to keep the cells growing at a fixed specific growth rate, often close to the maximum specific growth rate of the production strain. By maintaining dissolved oxygen above critical concentrations, the formation of byproducts is avoided. On the other hand, a constant feed of fresh substrate prevents starvation (Looser et al., 2014).

Under anaerobic or microaerobic conditions, C. glutamicum produces organic acids (Wieschalka et al., 2013). The goal of the fed-batch strategy is to maximize the amount of glucose fed, and consequently maximize biomass/product formation, while assuring an aerobic metabolism. The growth rate selected for the feeding calculation should be equal to or lower than the maximum value obtained in batch experiments, in order to avoid glucose accumulation. Based on the previous results, a fed-batch strategy with an initial glucose concentration in the batch phase of $40 \mathrm{~g} \mathrm{~L}^{-1}$ was followed to avoid anaerobic metabolism. Results from the batch experiments showed that after $14 \mathrm{~h}$ of fermentation, the glucose and biomass concentrations were $15 \mathrm{~g} \mathrm{~L}^{-1}$ and $12 \mathrm{~g} \mathrm{~L}^{-1}$, respectively. These were the conditions at which the feeding process was initiated. The feeding profile was defined based on the typical equation for exponential feeding (Eq. (1)) assuming a yield and specific growth rate similar to the ones obtained during the batch fermentations. 

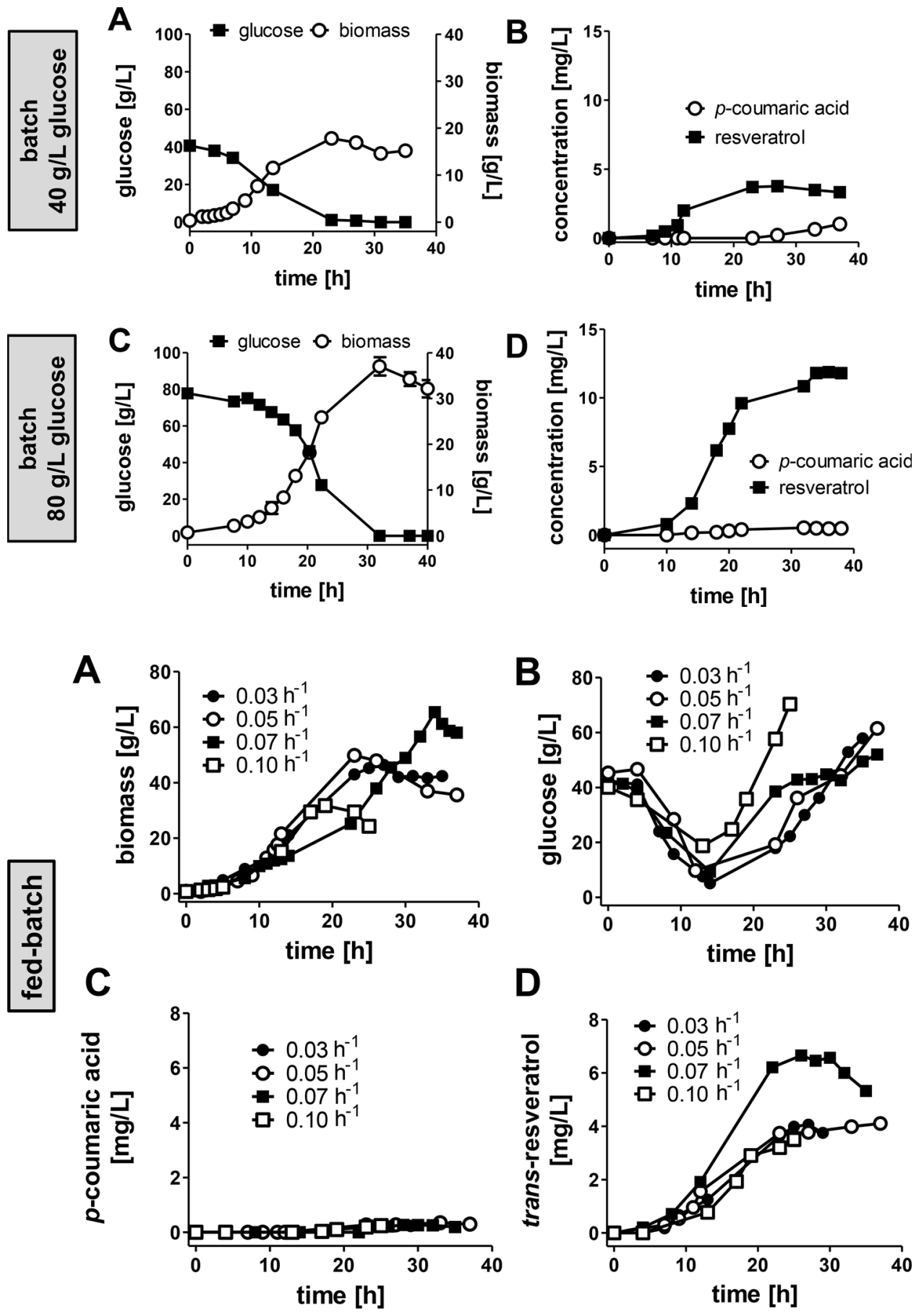

Fig. 2. Cell growth and resveratrol production with engineered C. glutamicum in batch experiments. A and $\mathrm{C}$ : biomass concentration and glucose consumption profile, B and D: resveratrol and p-coumaric acid concentrations. Data represent average values and standard deviation of two independent experiments. If no error bars are visible, the standard deviations were too small to be displayed.
Fig. 3. Cell growth and resveratrol production with engineered $C$. glutamicum in fed-batch experiments. Biomass concentration (A), glucose concentration (B), p-coumaric acid concentration (C) and resveratrol concentration (D) were analysed during fedbatch fermentation with the indicated growth rates of $0.03 \mathrm{~h}^{-1}\left(F_{A}\right), 0.05 \mathrm{~h}^{-1}\left(F_{B}\right), 0.07 \mathrm{~h}^{-1}\left(F_{C}\right)$ and $0.10 \mathrm{~h}^{-1}\left(F_{D}\right)$. Data represent average values and standard deviation of two independent experiments. If no error bars are visible, the standard deviations were too small to be displayed.
When the feeding in the fed-batch cultivation was started the biomass concentration increased exponentially for all the conditions tested (Fig. 3A), as the substrate was fed to the system. Thereafter, the biomass concentration did not increase and the glucose concentration began to build up in the culture medium (Fig. 3B). The amount of glucose fed to the bioreactor was higher than C. glutamicum could consume, even at lower feeding rates. Probably, other nutrients were limiting cell growth after this point. Fed-batch fermentation led to reasonably higher cell densities when compared to the batch results, while avoiding oxygen limitation. Previous results showed that maintaining an oxygen concentration higher than $30 \%$ in these bioreactors was necessary to guarantee an aerobic metabolism without the formation of organic acids (Oliveira et al., 2015). The production of organic acids (mainly lactate and succinate) was measured in all experimental conditions tested and was lower than $1.5 \mathrm{~g} \mathrm{~L}^{-1}$.

A biomass concentration of $65 \mathrm{~g} \mathrm{~L}^{-1}$ could be achieved after $34 \mathrm{~h}$ of cultivation (Fig. 3A), in the experiments with a feeding rate $F_{C}$. The amount of biomass obtained was 3.6-times higher than in batch cultivations with $40 \mathrm{~g} \mathrm{~L}^{-1}$ glucose and even 1.8-times higher when compared to a batch culture with $80 \mathrm{~g} \mathrm{~L}^{-1}$ glucose. Feeding rate $F_{C}$ proved to be the feeding rate that maximised both biomass and resveratrol concentrations. Feeding rate $F_{D}$ proved to be too high and the higher dilution rate caused by these conditions led to a decrease in the final product concentrations.

Despite the higher biomass production in the fed-batch fermentations, the highest resveratrol titer was only $7 \mathrm{mg} \mathrm{L}^{-1}$, for the experiment with a feeding rate $F_{C}$ (Fig. 3D). The respective yield of 0.04 (mg resveratrol per $g$ glucose) was much lower compared to the batch 
A

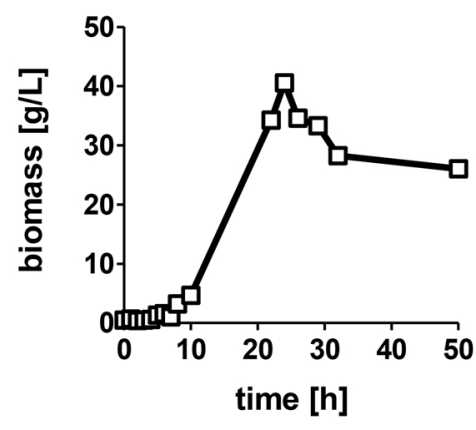

C

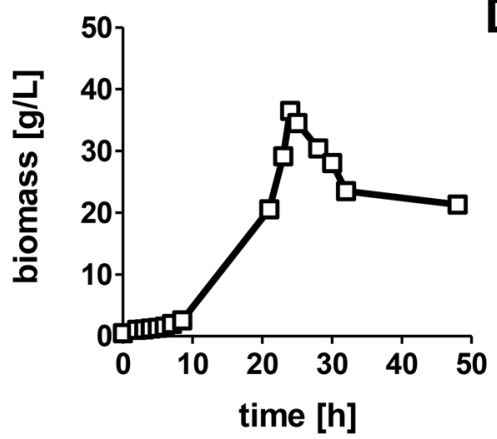

B

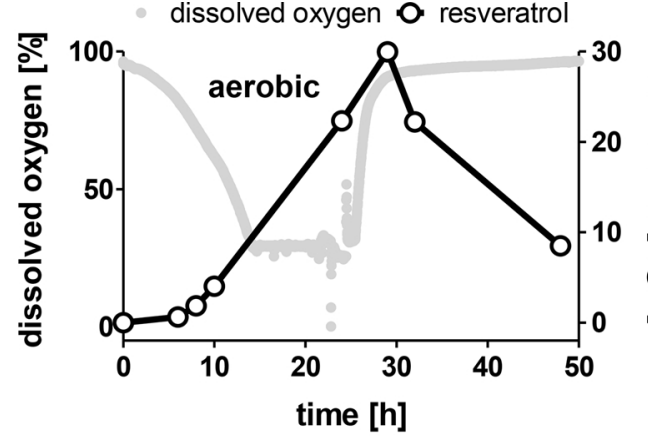

Fig. 4. Dissolved oxygen concentration, biomass and resveratrol production profiles of $C$. glutamicum. The cultivation was performed as batch fermentation with an initial glucose concentration of $80 \mathrm{~g} \mathrm{~L}^{-1}$ with an aerobic exponential phase and an aerobic stationary phase (A, B) or with an aerobic exponential phase and anaerobic conditions in the stationary phase (C, D). In figure D the dashed line indicates the start of nitrogen injection, which is the shifting moment between aerobic and anaerobic phases. Data represent average values and standard deviation of two independent experiments. If no error bars are visible, the standard deviations were too small to be displayed.
D

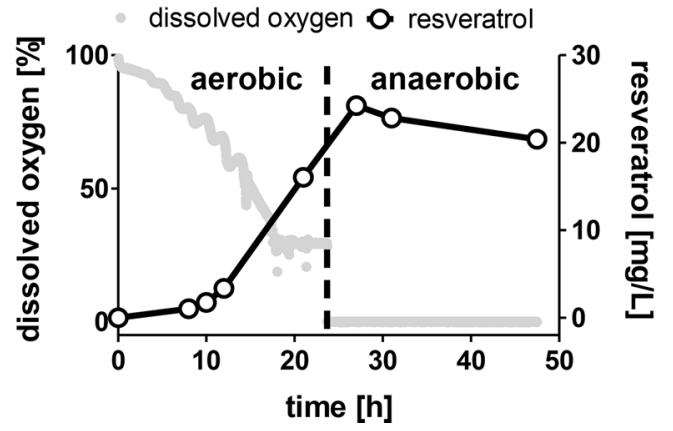

fermentations. In fact, the resveratrol production from glucose is a growth-coupled process and, for that reason, the yield was expected to be strongly correlated with the length of the growth phase. In batch experiments with a higher glucose concentration, cells grew at a lower specific growth rate when compared with the experiment performed at $40 \mathrm{~g} \mathrm{~L}^{-1}$, extending the exponential growth phase. These process conditions resulted in higher titers and yields. In the fed-batch experiments cells grew at an even lower specific growth rate and the overall glucose amount added was higher. Therefore, it is surprising that the resveratrol production is lower in the fed-batch fermentation mode. Probably, other effects influence the production of resveratrol during cultivation in fed-batch mode. One factor could be the specific growth rate value itself that, being too low, would decrease the malonyl-CoA pools, decreasing the fraction of the carbon flow directed towards resveratrol production. Another alternative explanation could be related to the energetic state of the cell that is expectedly different at different specific growth rates. At a lower specific growth rate the ATP maintenance becomes relatively more important in the cells, decreasing the energy that would be available for resveratrol production (Vos et al., 2015). In this scenario, we could speculate that there could be an optimum specific growth rate for resveratrol production below and above which product yields are affected.

At the end of the fed-batch fermentations, around $0.3 \mathrm{mg} \mathrm{L}^{-1}$ of $p$ coumaric acid accumulated in the exponential phase, for all the feeding rates tested (Fig. 3C). This observation reinforces the idea that the activity of the TAL enzyme is the controlling mechanism in the production pathway for trans-resveratrol in C. glutamicum (Kallscheuer et al., 2016b).

\subsection{High oxygen concentrations cause conversion of resveratrol during bioreactor cultivations}

To our surprise, we noticed that the resveratrol concentration decreased significantly after reaching a maximum product concentration (Fig. 2B and D). A decrease of $15-20 \%$ of the maximal resveratrol titer was observed during the stationary phase. This phenomenon did not occur during shake flask cultivations of the same strain before (data not shown) and was not so much visible during the batch cultivations with $80 \mathrm{~g} \mathrm{~L}^{-1}$ because these experiments were not kept for long enough in the stationary phase. In the platform strain C. glutamicum DelAro ${ }^{4}$ the genes coding for enzymes involved in the degradation of aromatic compounds are deleted, therefore any unspecific degradation by the host strain can be excluded. Instead, it is likely that a higher dissolved oxygen concentration in the bioreactor in this phase leads to product conversion. Previously, it could be shown that the resveratrol stability in solution is affected by high oxygen concentrations and light, as these factors can cause oxidation or multimerization of resveratrol which is related to its natural function as antioxidative compound (LucasAbellán et al., 2007; Trošt et al.,2009).

For further analysis of this phenomenon, we checked if product conversion is more pronounced in presence of higher resveratrol titers. To this end, the production strain was cultivated in CGXII medium with $80 \mathrm{~g} \mathrm{~L}^{-1}$ glucose and $5 \mathrm{mM}\left(0.82 \mathrm{~g} \mathrm{~L}^{-1}\right) p$-coumaric acid in the bioreactor. Under these conditions the strain reached a biomass concentration of $40 \mathrm{~g} \mathrm{~L}^{-1}$ (Fig. $4 \mathrm{~A}$ ) and produced $30 \mathrm{mg} \mathrm{L}^{-1}$ resveratrol after $30 \mathrm{~h}$ of cultivation (Fig. 4B). During the transition from the exponential phase to the stationary phase the dissolved oxygen concentration increased from $30 \%$ to $96 \%$ of saturation (Fig. 4B). Until the end of the experiment, $50 \mathrm{~h}$ after inoculation, the resveratrol titer dropped to $8 \mathrm{mg} \mathrm{L}^{-1}$, which is a decrease of $75 \%$ compared to the maximal titer (Fig. 4B). In a two-stage cultivation (aerobic exponential phase and anaerobic stationary phase) a very similar biomass concentration (Fig. 4C) and a maximal resveratrol concentration of $25 \mathrm{mg} \mathrm{L}^{-1}$ was reached after $30 \mathrm{~h}$ and more than $20 \mathrm{mg} \mathrm{L}^{-1}$ resveratrol could be still detected at the end of the cultivation (Fig. 4D). This experiment shows that high oxygen concentrations in the bioreactor are detrimental to the production of resveratrol. Until now, we were unable to detect any oxidation product of resveratrol and thus no additional information on the fate of resveratrol in the bioreactor can be provided at this stage. It is likely that the product is either an oxidation product or a resveratrol oligomer (Cichewicz et al., 2002; Yang et al., 2010).

We therefore assume that the observed decrease of the resveratrol concentration is indeed the result of spontaneous non-enzymatic conversion of resveratrol in the aerated fermenter and that the relatively high oxygen levels needed for cell growth can have a strong negative impact on the obtained resveratrol titers (maximal titer $30 \mathrm{mg} \mathrm{L}^{-1}$ dropped to $8 \mathrm{mg} \mathrm{L}^{-1}$ at the end of the cultivation). Thus, the determined product titers may not be a true reflection of the amount of 
resveratrol actually produced and this effect can be more pronounced in prolonged fermentations with high oxygen levels.

\section{Conclusions and outlook}

The obtained results show that there is a lot of potential to further improve resveratrol production with this $C$. glutamicum strain. An optimization of the resveratrol production in C. glutamicum should be performed from two different perspectives: (I) rational metabolic engineering of the host strain to improve the production capabilities (genetic engineering part) and (II) a detailed analysis and cautious optimization of the cultivation parameters. Strain engineering should address the current bottlenecks for production, which mainly refers to the low intracellular pool of malonyl-CoA and the insufficient TAL activity. For up-scaling of the production process, the oxygen-dependent conversion of resveratrol has to be avoided by choosing suitable process parameters. Furthermore, the effect of the specific growth rate in resveratrol accumulation should be further investigated. The combination of optimization efforts in both fields represents a necessary step towards an economically feasible production of resveratrol with C. glutamicum. We are currently addressing both strategies (strain construction and process optimization) in order to improve the production of resveratrol and other polyphenols based on the industrially relevant $C$. glutamicum.

\section{Acknowledgments}

We would like to thank the European Union Framework Program 7 "BacHBerry" (www.bachberry.eu), Project No. FP7- 613793 for financial support, the Portuguese Foundation for Science and Technology (FCT) under the scope of the strategic funding of UID/BIO/04469/2013 unit, COMPETE 2020 (POCI-01-0145-FEDER-006684) and BioTecNorte operation (NORTE-01-0145-FEDER-000004) funded by the European Regional Development Fund under the scope of Norte2020 - Programa Operacional Regional do Norte.

\section{References}

Baur, J.A., Sinclair, D.A., 2006. Therapeutic potential of resveratrol: the in vivo evidence. Nat. Rev. Drug Discov. 5, 493-506. http://dx.doi.org/10.1038/nrd2060.

Bavaresco, L., Petegolli, D., Cantù, E., Fregoni, M., Chiusa, G., Trevisan, M., 1997. Elicitation and accumulation of stilbene phytoalexins in grapevine berries infected by Botrytis cinerea. Vitis 36, 77-83.

Beekwilder, J., Wolswinkel, R., Jonker, H., Hall, R., De Rie Vos, C.H., Bovy, A., 2006. Production of resveratrol in recombinant microorganisms. Appl. Environ. Microbiol. 72, 5670-5672. http://dx.doi.org/10.1128/AEM.00609-06.

Buchholz, J., Schwentner, A., Brunnenkan, B., Gabris, C., Grimm, S., Gerstmeir, R., Takors, R., Eikmanns, B.J., Blombacha, B., 2013. Platform engineering of corynebacterium glutamicum with reduced pyruvate dehydrogenase complex activity for improved production of 1-lysine, l-valine, and 2-ketoisovalerate. Appl. Environ. Microbiol. 79, 5566-5575. http://dx.doi.org/10.1128/AEM.01741-13.

Cichewicz, R.H., Kouzi, S.A., Atta Ur, R., 2002. Resveratrol oligomers: Structure, chemistry, and biological activity. Stud. Nat. Prod. Chem. 26, 507-579.

Donnez, D., Jeandet, P., Clément, C., Courot, E., 2009. Bioproduction of resveratrol and stilbene derivatives by plant cells and microorganisms. Trends Biotechnol. 27, 706-713. http://dx.doi.org/10.1016/j.tibtech.2009.09.005.
Kallscheuer, N., Vogt, M., Kappelmann, J., Krumbach, K., Noack, S., Bott, M., Marienhagen, J., 2016a. Identification of the phd gene cluster responsible for phenylpropanoid utilization in Corynebacterium glutamicum. Appl. Microbiol. Biotechnol. 100, 1871-1881. http://dx.doi.org/10.1007/s00253-015-7165-1.

Kallscheuer, N., Vogt, M., Stenzel, A., Gätgens, J., Bott, M., Marienhagen, J., 2016b. Construction of a Corynebacterium glutamicum platform strain for the production of stilbenes and (2S)-flavanones. Metab. Eng. 38, 47-55. http://dx.doi.org/10.1016/j. ymben.2016.06.003.

Keilhauer, C., Eggeling, L., Sahm, H., 1993. Isoleucine synthesis in Corynebacterium glutamicum: Molecular analysis of the ilvB-ilvN-ilvC operon. J. Bacteriol. 175, 5595-5603.

Kiselev, K.V., 2011. Perspectives for production and application of resveratrol. Appl. Microbiol. Biotechnol. 90, 417-425. http://dx.doi.org/10.1007/s00253-011-3184-8.

Larisch, C., Nakunst, D., Hüser, A.T., Tauch, A., Kalinowski, J., 2007. The alternative sigma factor SigB of Corynebacterium glutamicum modulates global gene expression during transition from exponential growth to stationary phase. BMC Genomics 8, 4. http://dx.doi.org/10.1186/1471-2164-8-4.

Li, M., Kildegaard, K.R., Chen, Y., Rodriguez, A., Borodina, I., Nielsen, J., 2015. De novo production of resveratrol from glucose or ethanol by engineered Saccharomyces cerevisiae. Metab. Eng. 32, 1-11. http://dx.doi.org/10.1016/j.ymben.2015.08.007.

Lim, C.G., Fowler, Z.L., Hueller, T., Schaffer, S., Koffas, M.A.G., 2011. High-yield resveratrol production in engineered Escherichia coli. Appl. Environ. Microbiol. 77, 3451-3460. http://dx.doi.org/10.1128/AEM.02186-10.

Lin, J.A., Kuo, C.H., Chen, B.Y., Li, Y., Liu, Y.C., Chen, J.H., Shieh, C.J., 2016. A novel enzyme-assisted ultrasonic approach for highly efficient extraction of resveratrol from Polygonum cuspidatum. Ultrason. Sonochem. 32, 258-264. http://dx.doi.org/10. 1016/j.ultsonch.2016.03.018.

Looser, V., Bruhlmann, B., Bumbak, F., Stenger, C., Costa, M., Camattari, A., Fotiadis, D., Kovar, K., 2014. Cultivation strategies to enhance productivity of Pichia pastoris: A review. Biotechnol. Adv. 33, 1177-1193. http://dx.doi.org/10.1016/j.biotechadv. 2015.05.008.

Lucas-Abellán, C., Fortea, I., López-Nicolás, J.M., Núñez-Delicado, E., 2007. Cyclodextrins as resveratrol carrier system. Food Chem. 104, 39-44. http://dx.doi.org/10.1016/j. foodchem.2006.10.068.

Manach, C., Scalbert, A., Morand, C., Morand, C., Rémésy, C., Jiménez, L., 2004. Polyphenols: food sources and bioavailability. Am. J. Clin. Nutr. 79, 727-747. http:// dx.doi.org/10.1038/nature05488.

Marienhagen, J., Bott, M., 2013. Metabolic engineering of microorganisms for the synthesis of plant natural products. J. Biotechnol. 163, 166-178. http://dx.doi.org/ 10.1016/j.jbiotec.2012.06.001.

Oliveira, J.A., Braga, A., Rocha, I., Faria, N., 2015. The effect of hydrodynamic conditions in Corynebacterium glutamicum growth. MicroBiotec'15 - Congr. Microbiol. Biotechnol. 2015 (2014).

Shen, X.H., Zhou, N.Y., Liu, S.J., 2012. Degradation and assimilation of aromatic compounds by Corynebacterium glutamicum: Another potential for applications for this bacterium? Appl. Microbiol. Biotechnol. 95, 77-89. http://dx.doi.org/10.1007/ s00253-012-4139-4.

Trošt, K., Golc-Wondra, A., Prošek, M., 2009. Degradation of polyphenolic antioxidants in blueberry nectar aseptically filled in PET. Acta Chim. Slov. 56, 494-502.

Vos, T., de la, Torre, Cortés, P., van Gulik, W.M., Pronk, J.T., Daran-Lapujade, P., 2015. Growth-rate dependency of de novo resveratrol production in chemostat cultures of an engineered Saccharomyces cerevisiae strain. Microb. Cell Fact. 14, 133. http://dx doi.org/10.1186/s12934-015-0321-6.

Wieschalka, S., Blombach, B., Bott, M., Eikmanns, B.J., 2013. Bio-based production of organic acids with Corynebacterium glutamicum. Microb. Biotechnol. 6, 87-102. http://dx.doi.org/10.1111/1751-7915.12013.

Wu, J., Liu, P., Fan, Y., Bao, H., Du, G., Zhou, J., Chen, J., 2013. Multivariate modular metabolic engineering of Escherichia coli to produce resveratrol from l-tyrosine. J. Biotechnol. 167, 404-411. http://dx.doi.org/10.1016/j.jbiotec.2013.07.030.

Yang, Nae-Cherng, Lee, C.-H., Song, T.-Y., 2010. Evaluation of resveratrol oxidation in vitro and the crucial role of bicarbonate ions. Biosci. Biotechnol. Biochem. 74, 63-68. http://dx.doi.org/10.1271/bbb.90549.

van Summeren-Wesenhagen, P.V., Marienhagen, J., 2015. Metabolic engineering of Escherichia coli for the synthesis of the plant polyphenol pinosylvin. Appl. Environ. Microbiol. 81, 840-849. http://dx.doi.org/10.1128/AEM.02966-14. 